\section{Perfil das admissões em uma unidade de terapia intensiva obstétrica de uma maternidade brasileira}

\section{Admission profile in an obstetrics intensive care unit in a maternity hospital of Brazil}

Melania Maria Ramos de Amorim 1

Leila Katz 2

Marina Brito Ávila 3

Daniella Ericson Araújo 4

Mariana Valença 5

Carlos Japhet da Mata Albuquerque 6

Ana Rita Marinho Ribeiro Carvalho 7

Alex Sandro Rolland de Souza 8

\begin{abstract}
Objectives: to describe a three-year experience with obstetric Intensive Care Units (ICU), a unit allowing obstetricians to continue to care for critically ill obstetrics patients.

Methods: the study evaluated all admissions (933) to the Obstetric ICU, in the Instituto Materno Infantil Prof. Fernando Figueira (IMIP), from September 2002 to February 2005. Age, parity, diagnosis, admission time, diagnosis during ICU stay, associated complications, invasive procedures utilized, and final outcome were analyzed.

Results: hypertension (87\%), obstetric hemorrhage $(4.9 \%)$ and obstetric infection (2.1\%) were the major cause of the admissions analyzed. Mean age was 25 years, $65 \%$ of the patients delivered by cesarean-section. Anemia was a very common finding (58.4\%). Other diagnoses were renal insufficiency, thromboembolic disease, cardiac disease, acute pulmonary edema, sepsis, and hemorrhagic shock. Of the 814 patients with pregnancy-associated hypertension $65 \%$ had severe pre-ecclampsia, $16 \%$ mild preecclampsia, and $11 \%$ ecclampsia. HELLP syndrome was found in $46 \%$. Mechanical ventilation was necessary in $3.6 \%$ and hemotransfusion in $17 \%$ of the patients. Mean stay was five day (1 a 41) days. Death occurred in $2.4 \%$ of the patients.

Conclusions: the rate of deaths was low. An obstetric ICU managed by obstetricians could be a feasible way of dealing with maternal mortality.
\end{abstract}

Key words Intensive care unit, Obstetrics, Pregnancy, Hypertension, Critical care

\section{Resumo}

Objetivos: descrever a experiência de três anos com terapia intensiva em obstetrícia em Unidade de Terapia Intensiva em setor que permite que obstetras continuem conduzindo as pacientes obstétricas criticamente enfermas.

Métodos: estudo avaliando 933 pacientes atendidas na UTI obstétrica do Instituto Materno Infantil Prof. Fernando Figueira (IMIP) de setembro de 2002 a fevereiro de 2005. As variáveis foram idade, paridade, diagnóstico de admissão, época da admissão, diagnósticos e complicações durante o internamento, procedimentos invasivos empregados e resultado final.

Resultados: as três principais causas de internamento foram hipertensão (87\%), hemorragia obstétrica $(4,9 \%)$ e infecção (2,1\%). A idade média foi 25 anos $e$ $65 \%$ dos partos, cesarianas. Anemia foi achado freqüente $(58,4 \%)$. Outros diagnósticos: insuficiência renal, doença tromboembólica, cardiopatia, edema agudo de pulmão, sepse, choque hemorrágico. Das 814 pacientes admitidas com hipertensão associada à gestação, $65 \%$ tinham pré-eclâmpsia grave, $16 \%$ préeclâmpsia leve e $11 \%$ eclâmpsia. Síndrome HELLP ocorreu em 46\%. Ventilação mecânica foi necessária em $3,6 \%$ e hemotransfusão em $17 \%$. A duração média do internamento foi cinco dias (1-41). A taxa de óbito foi $2,4 \%$.

Conclusões: a taxa de morte foi relativamente baixa, sugerindo que uma UTI conduzida por obstetras pode ser uma estratégia factivel para reduzir a mortalidade materna.

Palavras-chave Unidades de terapia intensiva, Obstetrícia, Gravidez, Hipertensão, Cuidados críticos 


\section{Introdução}

A chance de uma mulher, durante o ciclo grávidopuerperal, ser admitida em uma Unidade de Terapia Intensiva (UTI) é bem maior do que a de uma mulher jovem, não-grávida. ${ }^{1}$ Estima-se que $0,1 \%$ a $0,9 \%$ das gestantes desenvolvem complicações, requerendo o internamento em Unidade de Terapia Intensiva, sendo as principais indicações hipertensão, hemorragia, insuficiência respiratória e sepse. ${ }^{2-5}$ Destacase, outrossim, que o prognóstico dessas pacientes em geral é bom, requerendo geralmente intervenção mínima, com baixas taxas de mortalidade, em geral inferior a $3 \% .3,6,7$

A interface clínico-obstétrica assume especial importância em se tratando de uma unidade de cuidados intensivos para pacientes no ciclo grávidopuerperal. Alterações fisiológicas próprias da gestação distinguem essas pacientes de outros adultos jovens. Diversas condições clínicas têm seu curso afetado pela gravidez, além do que varia a interpretação de testes diagnósticos e valores laboratoriais. Por outro lado, complicações exclusivas da gravidez podem não ser familiares aos clínicos, como a préeclâmpsia/eclâmpsia e a embolia do líquido amniótico. Dessa forma, os obstetras devem estar familiarizados com os princípios básicos da terapia intensiva, tanto para tratar as pacientes obstétricas como para atuar como consultores dentro do time de intensivistas. 1

A proposta de um modelo de UTI voltada exclusivamente para o tratamento de pacientes obstétricas, ao invés de se operar com a transferência dessas para unidades gerais de terapia intensiva, não é recente. Em 1990, Mabie e Sibai2 descreveram os resultados da implementação de uma UTI obstétrica na Universidade do Tennessee, em Memphis, identificando os seguintes benefícios: a) reconhecimento e tratamento precoce das complicações, em decorrência da observação intensiva; b) progressiva aquisição de habilidade e experiência com a monitorização intensiva pela equipe médica, garantindo o tratamento imediato, racional e adequado das pacientes hemodinamicamente estáveis; c) melhora da continuidade da assistência, tanto antes como depois do parto; d) importante treinamento e aprendizagem tanto dos residentes como dos médicos do staff em terapia intensiva, bem como no tratamento de complicações médicas raras associadas à gravidez. Os autores concluíram que não apenas as gestantes com condição clínica grave podem ser conduzidas com sucesso em uma UTI obstétrica, mas que a Terapia Intensiva representa indubitavelmente uma parte da prática obstétrica, devendo ser incorporada ao programa de treinamento e formação de especialistas.

$\mathrm{Na}$ Maternidade do Instituto Materno Infantil Prof. Fernando Figueira (IMIP) foi implantada em 2002 uma UTI Obstétrica, sendo todos os plantonistas obstetras de formação, com treinamento específico em terapia intensiva.

Reconhecendo a necessidade de avaliar a experiência que esse serviço vem progressivamente adquirindo com a implementação da UTI Obstétrica, foi realizado o presente estudo que tem como objetivos de determinar a freqüência de admissões em relação ao total de partos atendidos no serviço, identificar as principais causas de internamento e definir o perfil clínico e o prognóstico das pacientes admitidas na UTI.

\section{Métodos}

Realizou-se um estudo observacional, longitudinal, do tipo coorte na UTI Obstétrica do IMIP, unidade destinada ao atendimento às complicações graves próprias da gestação ou nela intercorrentes. Essa UTI conta com 12 leitos, dos quais seis são equipados com respirador. Os plantonistas são todos médicos-obstetras com treinamento em terapia intensiva, sob a supervisão de um cardiologista e um clínico (diaristas), tendo ainda a participação de cirurgiões de sobreaviso. Especialistas como neurologistas e nefrologistas dão apoio em regime de interconsultas e pareceres, e todos os procedimentos de imagem (raios-X, ultra-sonografia, tomografia computadorizada) são realizados dentro da própria instituição.

O período do estudo foi de setembro de 2002 a fevereiro de 2005 , período no qual todas as pacientes atendidas na UTI totalizaram 2547.

O cálculo do tamanho da amostra resultou em 933 pacientes e foi realizado prevendo-se uma frequiência do principal desfecho (morte materna) de $2,5 \%$, com uma precisão de $1 \%$ e nível de confiança de $5 \%$. Sortearam-se os prontuários a partir dos registros no livro de internamento da UTI. O estudo foi previamente aprovado pelo Comitê de Ética em Pesquisa da Instituição.

Analisaram-se as seguintes variáveis: época de internamento (fase do ciclo grávido-puerperal), motivo do internamento na UTI (diagnóstico de admissão), idade, paridade, procedência, escolaridade, idade gestacional no parto, tipo do parto, diagnósticos e complicações durante o internamento (incluindo óbito), procedimentos invasivos (incluindo ventilação mecânica) e duração do internamento. 
Tanto as indicações de internação em unidade de terapia intensiva como o acompanhamento das pacientes internadas seguiram as rotinas preconizadas no Manual de Normas da UTI Obstétrica da instituição, ${ }^{8}$ de acordo com o diagnóstico e as complicações associadas.

Na análise dos dados utilizou-se o programa EpiInfo 3.3.2 para Windows. Construíram-se tabelas de distribuição de freqüência para as variáveis categóricas, calculando-se ainda médias e seus respectivos desvios-padrão para as variáveis quantitativas.

\section{Resultados}

Durante o período do estudo, houve 2547 admissões na UTI obstétrica e 15.687 ocorrências obstétricas no IMIP. As admissões em UTI corresponderam, portanto, a 16,2\% das ocorrências no período. Das 933 pacientes estudadas, $12 \%$ foram admitidas durante a gravidez, $87 \%$ no pós-parto e $1,0 \%$ depois de abortamento ou prenhez ectópica (Tabela 1). As principais causas de internação foram hipertensão, hemorragia e infecção no ciclo grávido-puerperal. As síndromes hipertensivas corresponderam a $87 \%$ dos diagnósticos de admissão, seguindo-se as

\section{Tabela 1}

Distribuição das gestantes conforme a época de admissão

\begin{tabular}{lrr}
\hline & $\mathbf{N}$ & $\%$ \\
\hline Durante a gravidez & 116 & 12,4 \\
Pós-parto & 808 & 86,6 \\
Pós-abortamento / prenhez ectópica & 9 & 1,0 \\
Total & 933 & 100,0 \\
\hline
\end{tabular}

Tabela 2

Principais causas de internamento na Unidade de Terapia Intensiva Obstétrica

\begin{tabular}{lrr}
\hline Causa de internamento & N & $\%$ \\
\hline Síndromes hipertensivas da gravidez & 814 & 87,2 \\
Síndromesh hemorrágicas da gravidez & 46 & 4,9 \\
Infecção puerperal & 20 & 2,1 \\
Outras infecções & 27 & 2,9 \\
Outras causas & 26 & 2,8 \\
Total & 933 & 100,0 \\
\hline
\end{tabular}

Tabela 3

Características das pacientes internadas na Unidade de Terapia Intensiva Obstétrica do Instituto Materno Infantil Prof. Fernando Figueira, IMIP. Recife, Pernambuco.

\begin{tabular}{|c|c|c|}
\hline Características & $\mathbf{N}$ & $\%$ \\
\hline \multicolumn{3}{|l|}{ Idade } \\
\hline 10-19 anos & 232 & 24,9 \\
\hline 20-34 anos & 597 & 64,0 \\
\hline$\geq 35$ anos & 104 & 11,1 \\
\hline \multicolumn{3}{|l|}{ Procedência } \\
\hline Recife e Região Metropolitana & 485 & 52,1 \\
\hline Outras cidades de Pernambuco & 381 & 40,6 \\
\hline Cidades de outros estados & 57 & 6,1 \\
\hline \multicolumn{3}{|l|}{ Escolaridade } \\
\hline $0-3$ anos & 126 & 13,5 \\
\hline $4-7$ anos & 363 & 38,8 \\
\hline $8-10$ anos & 249 & 26,7 \\
\hline 11 ou mais & 128 & 13,8 \\
\hline \multicolumn{3}{|l|}{ Paridade } \\
\hline Nulíparas & 247 & 27,3 \\
\hline Um parto & 341 & 37,6 \\
\hline Dois ou mais partos & 318 & 45,1 \\
\hline \multicolumn{3}{|l|}{ Idade gestacional no parto * } \\
\hline$<32$ semanas & 93 & 10,7 \\
\hline $32-36$ semanas & 384 & 44,0 \\
\hline$\geq 37$ semanas & 396 & 45,3 \\
\hline \multicolumn{3}{|l|}{ Tipo de parto * } \\
\hline Vaginal & 302 & 34,6 \\
\hline Cesárea & 571 & 65,4 \\
\hline
\end{tabular}

* Incluídas somente as pacientes obstétricas com nascimento de concepto vivo ou morto a partir de 22 semanas e/ou peso $\geq 500 \mathrm{~g}$.

Tabela 4

Principais diagnósticos e complicações durante a internação na Unidade de Terapia Intensiva, Obstétrica do Instituto Materno Infantil Prof. Fernando Figueira, IMIP. Recife, ernambuco.

\begin{tabular}{lrr}
\hline Diagnóstico/Complicação & N & $\%$ \\
\hline Síndromes Hipertensivas da Gravidez & 829 & 88,9 \\
Anemia & 545 & 58,4 \\
Choque hemorrágico & 82 & 8,8 \\
Cardiopatia & 51 & 5,5 \\
Sepse & 26 & 2,8 \\
Síndrome do Desconforto Respiratório do Adulto & 15 & 1,6 \\
Edema agudo de pulmão & 14 & 1,5 \\
Doença tromboembólica & 12 & 1,3 \\
Insuficiência renal aguda & 10 & 1,1 \\
Anemia falciforme & 2 & 0,2 \\
Cetoacidose diabética & 2 & 0,2 \\
Disfunção de múltiplos órgãos & 2 & 0,2 \\
Óbito & 22 & 2,4 \\
\hline
\end{tabular}


Tabela 5

Formas clínicas das síndromes hipertensivas Unidade de Terapa Intensiva Obstétrica do Instituto Materno Infantil Prof. Fernando Figueira, IMIP. Recife, Pernambuco.

\begin{tabular}{lrr}
\hline Distúrbios hipertensivos & N & $\%$ \\
\hline Pré-eclâmpsia leve & 132 & 15,9 \\
Pré-eclâmpsia grave & 540 & 65,1 \\
Eclâmpsia & 92 & 11,1 \\
HAS crônica & 5 & 0,6 \\
Pré-eclâmpsia superposta & 44 & 5,3 \\
Hipertensão gestacional & 16 & 1,9 \\
Síndrome HELLP & 380 & 45,8 \\
$\quad$ Síndrome HELLP completa & 58 & 7,0 \\
Síndrome HELLP incompleta & 322 & 38,8 \\
\hline
\end{tabular}

HELLP = Hemolysis-Elevated Liver enzymes-Low Platelet count syndrome"

Tabela 6

Procedimentos invasivos* realizados na Unidade de Terapa Intensiva Obstétrica do Instituto Materno Infantil Prof. Fernando Figueira, IMIP. Recife, Pernambuco.

\begin{tabular}{lrr}
\hline Procedimentos invasivos & $\mathbf{n}$ & $\%$ \\
\hline Hemotransfusão & 160 & 17,1 \\
Punção venosa ventral & 39 & 4,2 \\
Ventilação mecânica & 34 & 3,6 \\
Toracocentese/drenagem torácica & 33 & 3,5 \\
Uso de drogas vasoativas & 9 & 1,0 \\
Dissecção venosa & 5 & 0,5 \\
Hemodiálise & 4 & 0,4 \\
Reanimação cardiopulmonar & 2 & 0,2 \\
Traqueostomia & 1 & 0,1 \\
\hline
\end{tabular}

* Uma paciente pode ter sido submetida a mais de um procedimento invasivo.

\section{Tabela 7}

Duração do internamento* na UTI Unidade de Terapa Intensiva Obstétrica do Instituto Materno Infantil Prof. Fernando Figueira, IMIP. Recife, Pernambuco.

\begin{tabular}{lrr}
\hline Duração do internamento (em dias) & $\mathbf{n}$ & $\%$ \\
\hline 1 & 74 & 7,9 \\
$2-4$ & 446 & 47,8 \\
$5-9$ & 325 & 34,8 \\
$10-14$ & 47 & 5,1 \\
$>15$ & 41 & 4,4 \\
Variação $(1-41)$ & - & - \\
\hline
\end{tabular}

* Foram analisados apenas os casos que obtiveram alta, excluindo-se os óbitos. síndromes hemorrágicas $(4,9 \%)$ e a infecção puerperal $(2,1 \%)$ (Tabela 2$)$. A média de idade foi de 25 anos $(24,8 \pm 6,9)$, sendo que a maior parte das pacientes $(64 \%)$ encontrava-se na faixa etária entre 20 e 34 anos. Cerca de $53 \%$ procediam de Recife e Região Metropolitana, e $47 \%$ de cidades do interior do estado de Pernambuco ou outros estados. Em torno de $52 \%$ tinham escolaridade entre zero e sete anos de estudo, $48 \%$ tinham oito ou mais anos completos de estudo. Em relação à paridade, $27 \%$ eram primíparas e $38 \%$ multíparas. O parto prematuro (abaixo de 37 semanas) ocorreu em 55\% dos casos. O tipo de parto mais freqüente foi a cesariana, realizada em $65 \%$ das pacientes (Tabela 3 ).

Analisando-se os principais diagnósticos e complicações durante a admissão, verificou-se um predomínio das síndromes hipertensivas da gravidez $(88,9 \%)$, anemia $(58,4 \%)$, choque hemorrágico $(8,8 \%)$, cardiopatia $(5,5 \%)$ e sepse $(2,8 \%)$. Outros diagnósticos, menos freqüentes, corresponderam a Síndrome do Desconforto Respiratório do adulto $(1,6 \%)$, edema agudo de pulmão $(1,5 \%)$, doença tromboembólica $(1,3 \%)$, insuficiência renal aguda $(1,1 \%)$, anemia falciforme $(0,2 \%)$, cetoacidose diabética $(0,2 \%)$ e disfunção de múltiplos órgãos $(0,2 \%)$. A taxa de óbitos foi de $2,4 \%$ (Tabela 4 ).

Quanto às diversas formas clínicas das síndromes hipertensivas, observou-se $65 \%$ de casos de pré-eclâmpsia grave, $16 \%$ de casos de préeclâmpsia leve, $11 \%$ de eclâmpsia, $5 \%$ de préeclâmpsia superposta, $2 \%$ de hipertensão gestacional e $0,6 \%$ de hipertensão arterial sistêmica crônica. A síndrome HELLP foi diagnosticada em $46 \%$ desses casos de hipertensão e gravidez, com $7 \%$ de síndrome HELLP completa e $39 \%$ de síndrome HELLP incompleta (Tabela 5).

Em relação aos procedimentos invasivos, foram realizadas hemotransfusão em $17 \%$, punção venosa central em $4 \%$, ventilação mecânica em 3,6\%, toracocentese/drenagem torácia em $3,5 \%$, uso de drogas vasoativas em $1 \%$, dissecção venosa em $0,5 \%$, hemodiálise em $0,4 \%$, reanimação cardiopulmonar em $0,2 \%$ e traqueostomia em $0,1 \%$ dos casos (Tabela 6 ).

A duração do internamento (entre as pacientes que tiveram alta) variou entre um e 41 dias, com média em torno de cinco dias $(5,13 \pm 4,28)$. Aproximadamente torno de $56 \%$ das pacientes tiveram alta da UTI com menos de cinco dias e apenas $4,4 \%$ permaneceram internadas por mais de duas semanas (Tabela 7). 


\section{Discussão}

No presente estudo foi observada uma elevada freqüência de admissões na UTI obstétrica, correspondendo a $16 \%$ do total de ocorrências no serviço. Apesar de nos países desenvolvidos a necessidade relatada de internamentos em UTI por pacientes obstétricas ser baixa, esses países têm, caracteristicamente, assistência pré-natal eficiente e baixas taxas de mortalidade materna. ${ }^{9}$ Em países em desenvolvimento, como a mortalidade materna é mais elevada, há estudos que mostram que até $10 \%$ das admissões em unidades de terapia intensiva geral são representadas por pacientes no ciclo grávidopuerperal. 9

Por outro lado, essa taxa elevada de internação pode ser explicada pelo fato de que, entre os critérios de admissão à UTI obstétrica do Centro de Atenção à Mulher $(\mathrm{CAM})$ do IMIP está a profilaxia da eclâmpsia com sulfato de magnésio, inclusive em casos de pré-eclâmpsia leve. As síndromes hipertensivas constituem, de fato, a complicação mais frequiente em gestantes internadas no IMIP e evidências nível I corroboram o uso do sulfato de magnésio para prevenção das convulsões em pacientes com pré-eclâmpsia. 10

A maioria das pacientes foi internada no pósparto (87\%), o que é compatível com a literatura, que demonstra maior freqüência de internamentos para terapia intensiva no puerpério.3,11,12 Como possíveis explicações, são sugeridas, a perda sanguínea que ocorre no parto, levando a descompensação hemodinâmica em pacientes que antes mantinham um frágil equilíbrio, e o adiamento da transferência das pacientes para UTI.13

As principais causas de internação foram hipertensão (87\%), hemorragia $(4,9 \%)$ e infecção no ciclo grávido-puerperal $(2,1 \%)$. Resultados semelhantes foram descritos em um estudo italiano, ${ }^{4}$ no qual $70 \%$ das internações relacionaram-se às síndromes hipertensivas da gravidez. Em um estudo brasileiro, ${ }^{14}$ as principais causas de internamento em unidades de terapia intensiva foram pré-eclâmpsia, hemorragia e sepse.

Em relação às síndromes hemorrágicas como causa de internamento em UTI, vale destacar que essas são mais comuns em países sem uma boa estrutura de bancos de sangue, como na Índia, em que as síndromes hemorrágicas pós-parto são responsáveis por $24 \%$ das admissões obstétricas. 15

As complicações e mortes por hemorragia são características da falta de assistência adequada ao parto e ao pós-parto imediato, sendo mais elevadas nos países menos desenvolvidos e mais comuns em mulheres pobres e com maior paridade. 16 A assistência ao parto assume um papel extremamente relevante, porquanto a maioria das mortes irá ocorrer no período periparto, quando podem acontecer os eventos cataclísmicos, e é essencial a intervenção de profissionais qualificados e hospitais bem aparelhados para o tratamento das emergências obstétricas.

A média de idade encontrada no presente estudo (25 anos) foi semelhante à descrita por diversos autores, 15 o que muito provavelmente decorre do fato de que a clientela da UTI obstétrica é constituída por mulheres na fase reprodutiva.

Em relação à paridade, conquanto tenhamos admitido $27 \%$ das pacientes no curso da primeira gestação, essa taxa ainda é baixa considerando-se as informações disponíveis na literatura. Em um estudo indiano foram avaliados fatores prognósticos em pacientes admitidas em unidades de terapia intensiva, 46,8\% de primigrávidas. 15 No Reino Unido, $50 \%$ das admissões em UTI obstétricas foram de primigrávidas. 3 Como não comparamos a frequiência de primigestas internadas na UTI com a freqüência global no serviço, fica difícil interpretar esse resultado, mas é possível que muitas gestações de risco ocorram em multíparas sem acesso adequado ao planejamento familiar e a cuidados pré-natais. 17,18

$\mathrm{O}$ parto prematuro foi bastante freqüente $(55 \%$ dos casos), podendo ser explicado pelo fato de muitas pacientes terem já sido submetidas à interrupção da gestação por morbidades próprias da gravidez, como a pré-eclâmpsia, que pode ocorrer em idade gestacional precoce, ou por descompensação de doenças clínicas pré-existentes.

O tipo de parto mais frequiente foi a cesariana, realizada em cerca de dois terços, fato que é justificado em parte pela gravidade das complicações, impondo, em geral, o término da gestação em fase precoce, com condições cervicais desfavoráveis e/ou comprometimento do bem-estar fetal. Taxas de cesariana iguais ou superiores a $70 \%$ são descritas na literatura, 6 e na Ásia já foram relatadas taxas de até $95 \% .5$ Destaca-se, outrossim, que uma parcela significativa das pacientes foi transferida de outros serviços já depois do término da gravidez por via alta, associando-se ao quadro clínico grave a morbidade característica da operação cesariana. 19

A taxa de óbitos encontrada $(2,4 \%)$ aproximouse daquela descrita na literatura por autores canadenses e americanos (2-4\%),2,3,7,11 em contraste com a alta mortalidade encontrada em estudos indianos $(21,8 \%), 15$ em um estudo brasileiro $(33,8 \%)^{14} \mathrm{e}$ a elevadíssima mortalidade relatada em um estudo africano $(60 \%) .20$

A frequência de hemotransfusão no presente 
estudo foi de $17 \%$, mais baixa do que a encontrada em um estudo indiano, em que hemotransfusão de concentrado de hemácias foi necessária em 38,2\% e de plasma e concentrado de plaquetas em $29,6 \% .15$ Essa diferença pode ser explicada pela elevada freqüência de hemorragia na Índia $(17,2 \%)$, bem superior à encontrada em nosso estudo.

De qualquer forma, a freqüência global de procedimentos invasivos na UTI obstétrica do IMIP foi baixa, sendo a punção venosa central necessária em $4 \%$, a ventilação mecânica em $3,6 \%$, a toracocentese/drenagem torácica em 3,5\%, o uso de drogas vasoativas em $1 \%$, a dissecção venosa em $0,5 \%$ e a hemodiálise em $0,4 \%$. Em estudo realizado na Índia, a necessidade de drogas inotrópicas foi de $18,5 \%$, enquanto a ventilação mecânica foi realizada em $18,5 \%$ e a diálise em $10,4 \%$ das pacientes, 15 possivelmente caracterizando uma pior condição das mesmas no momento da admissão à unidade de terapia intensiva.

O perfil das pacientes admitidas e as próprias características de uma UTI obstétrica também justificam a diferença em relação a outros estudos: no Reino Unido, um estudo avaliou as pacientes no ciclo grávido-puerperal admitidas em unidades de terapia intensiva tanto clínicas como cirúrgicas, encontrando necessidade de ventilação mecânica em $45 \%$ dos casos, uso de drogas inotrópicas em $19 \%$ e hemodiálise em 3\%.3 A disponibilidade de uma UTI específica para pacientes obstétricas facilita a internação precoce, antes da deterioração grave ou fatal da condição clínica.

Em um estudo britânico, 3 foram avaliadas as pacientes admitidas em terapia intensiva por hipertensão associada à gestação e foi observado que essas pacientes tinham menor probabilidade de necessitar de procedimentos invasivos que pacientes admitidas com outros diagnósticos (35 x 48\%). Como a maioria de nossas pacientes $(87 \%)$ tinha diagnóstico de alguma forma clínica das síndromes hipertensivas, predominando a pré-eclâmpsia, seria realmente de se esperar uma menor necessidade de procedimentos invasivos.

A duração do internamento (entre as pacientes que tiveram alta) variou entre um e 41 dias, com média em torno de cinco dias. Essa média de duração do internamento foi mais alta do que a relatada por alguns autores do Reino $\mathrm{Unido}^{3}$ que encontraram um tempo de internamento médio de um dia, porém se aproximou dos dados encontrados por outros autores, 15 como no estudo realizado na Índia, com média de internamento em torno de cinco dias. Essa duração reflete, possivelmente, o tempo necessário para recuperação pós-parto das doenças obstétricas como pré-eclâmpsia/eclâmpsia, síndrome HELLP e esteatose hepática aguda da gestação. ${ }^{21}$

Em 1998, Mantel et al. 22 descreveram o conceito de near miss maternal mortality referindo-se àqueles casos de pacientes no ciclo grávido-puerperal com disfunção ou falha orgânica, que morreriam caso não se realizassem tratamentos ou medidas de suporte, ou se esses fossem dados de forma inadequada. Os critérios considerados por Mantel como near-miss assemelham-se bastante aos critérios de internamento em UTI obstétrica adotados no IMIP. Trata-se de pacientes instáveis que, com cuidados intensivos, recuperam a estabilidade e "escapam" das estatísticas de mortalidade em função desses cuidados.

Estudos mostram que a verdadeira magnitude da morbidade materna é maior do que a observada pelo índice bruto de mortalidade. Apesar de se descrever uma baixa prevalência das morbidades específicas que atingem mulheres nesse período, o fardo da morbidade é alto, sendo na grande maioria das vezes prevenível. 23

$\mathrm{O}$ esquema a seguir sugere um continuum que uma paciente percorreria desde um ponto em que se encontra completamente saudável até o extremo onde ocorreria a morte materna:

GRAVIDEZ » NORMAL/SAUDÁVEL 》 MORBIDADE 》 MORBIDADE GRAVE » NEAR-MISS » MORTE MATERNA.

Esse continuum poderia ser interrompido em vários pontos através da adoção de medidas preventivas em diversos níveis (primário, secundário, terciário). Evidências convincentes demonstram que pelo menos metade das mortes maternas seriam preveníveis por mudanças nas pacientes, nos prestadores de saúde e do sistema de saúde. 24 Evidentemente, nem todas as mortes maternas podem ser prevenidas, mas as gritantes disparidades entre os países desenvolvidos e em desenvolvimento apontam para a possibilidade de redução da razão de mortalidade materna. ${ }^{16}$

Um estudo estimou que, caso fossem tomadas todas as medidas de prevenção cabíveis no intuito de modificar a progressão desse continuum, menos $41 \%$ das mulheres teriam morrido e menos $45 \%$ teriam só morbidade grave, ao invés de near-miss. 25

A proposta de uma unidade de terapia intensiva obstétrica insere-se exatamente nesse contexto, uma vez que urgências e emergências obstétricas podem ocorrer mesmo quando as medidas gerais são adotadas, incluindo a melhora do nível socioeconômico e da escolaridade da população e à adequada assistência durante o pré-natal, parto e puerpério. Há que se garantir o tratamento adequado dessas condições, evitando complicações mais graves e o óbito. 1 


\section{Conclusões}

A hipertensão associada à gestação foi a maior causa de internamento na UTI obstétrica. A despeito da causa, houve uma elevada freqüência de admissões, correspondendo a $16 \%$ do total de ocorrências no serviço. O parto prematuro (abaixo de 37 semanas) foi bastante freqüente ( $55 \%$ dos casos) e, independentemente da idade gestacional, o tipo de parto mais realizado foi a cesariana, em $65 \%$ das pacientes.

A freqüência global de procedimentos invasivos na UTI obstétrica do IMIP foi baixa, assim como a taxa de morte $(2,4 \%)$, sugerindo que uma UTI conduzida por obstetras pode ser uma estratégia factível para reduzir a mortalidade materna.

\section{Referências}

1. Gabbe S. Obstetrics; normal and problem pregnancies. 4 . ed. London: Churchill-Livingstone; 2002.

2. Mabie WC, Sibai BM. Treatment in an obstetric intensive care unit. Am J Obstet Gynecol. 1990; 162: 1-4.

3. Hazelgrove IF, Price C, Pappachan VJ, Smith JB. Multicenter study of obstetric admissions to 14 intensive care units in southern England. Crit Care Med. 2001; 29: 770-775.

4. Loverro G, Pansini V, Greco P, Vimercati A, Parisi AM, Selvaggi L. Indications and outcome for intensive care unit admission during puerperium. Arch Gynecol Obstet. 2001; 265:195-8.

5. Quah TC, Chiu JW, Tan KH, Yeo SW, Tan HM. Obstetric admissions to the intensive therapy unit of a tertiary care institution. Ann Acad Med Singapore. 2001; 30: 250-3.

6. Panchal S, Arria AM, Harris AP. Intensive care utilization during hospital admission for delivery: prevalence, risk factors, and outcomes in a statewide population. Anesthesiology. 2000; 92: 1537-44.

7. Afessa B, Green B, Delke I, Koch K. Systemic inflammatory response syndrome, organ failure, and outcome in critically ill obstetric patients treated in an ICU. Chest. 2001; 120:1271-7.

8. Santos LC, Amorim MMR, Katz L, Albuquerque CJM. Terapia intensiva em obstetrícia. São Paulo: MEDSI; 2004.

9. Munnur U, Guntupalli KK. Obstetric patients requiring intensive care unit admission Crit Care Med. 2004; 32: 1418-9.

10. The Magpie Trial Collaboration Group. Do women with pre-eclampsia, and their babies, benefit from magnesium sulphate? The Magpie Trial: a randomised placebocontrolled trial. Lancet. 2002; 359 1877-90.

11. Zeeman GG, Wendel GDJr, Cunningham FG. A blueprint for obstetric critical care. Am J Obstet Gynecol. 2003; 188: 532-6.
Há ainda muito a aprender em relação aos cuidados intensivos que norteiam as pacientes no ciclo grávido-puerperal. A UTI obstétrica do IMIP visa contribuir continuamente nesse sentido, almejando um objetivo maior que é a redução da morbimortalidade dessas pacientes.

\section{Agradecimentos}

Ao Instituto Materno Infantil Prof. Fernando Figueira, IMIP pelo suporte e ao Conselho Nacional de Desenvolvimento Científico e Tecnológico, CNPQ pela bolsa do Programa de Iniciação Científica.
12. Karnad DR, Guntupalli KK. Critical illness and pregnancy: review of a global problem. Crit Care Clin. 2004; 20: 55576.

13. Kilpatrick SJ, Matthay MA. Obstetric patients requiring critical care: a five-year review. Chest. 1992; 101: 1407-12.

14. Dias de Souza JP, Duarte G, Basile-Filho A. Near-miss maternal mortality in developing countries. Eur J Obstet Gynecol Reprod Biol. 2002; 104: 80

15. Karnad DR. Prognostic factors in obstetric patients admitted to an Indian intensive care unit. Crit Care Med. 2004; 32: 1294-99.

16. WHO (World Health Organization), UNICEF (United Nations International Children's Emergency Fund). Revised 1990 estimates of maternal mortality. A new approach by WHO and UNICEF. Geneva; 1996.

17. Cecatti JG, Faúndes A, Surita FGC. Maternal mortality in Campinas: evolution, under-registration and avoidance. Rev Paul Med. 1999; 117: 5-12.

18. Costa AAR, Ribas MSSS, Amorim MMR, Santos LC. Mortalidade materna na cidade do Recife. Rev Bras Ginecol Obstet. 2002; 24: 455-62.

19. Jackson N, Paterson-Brown S. Physical sequelae of caesarean section. Best Pract Res Clin Obstet Gynaecol. 2001; 15: 49-61.

20. Dao B, Rouamba A, Ouedraogo D. Transfer of obstetric patients to an intensive care unit: an eighty-two case report in Burkina Faso. Gynecol Obstet Fertil. 2003; 31: 123-6.

21. Norwitz ER, Hsu CD, Repke JT. Acute complications of pre-eclampsia. Clin Obstet Gynecol. 2002; 45: 308-29.

22. Mantel GD, Buchmann E, Rees H, Pattinson RC. Severe acute maternal morbidity: a pilot study of a definition for a near-miss. Br J Obstet Gynaecol. 1998; 105: 985-90.

23. Danel I, Berg C, Johnson CH, Atrash H. Magnitude of maternal morbidity during labor and delivery: United States, 1993-1997. Am J Public Health. 2003; 93: 631-4. 
24. Nannini A, Weiss J, Goldstein R, Fogerty S. Pregnancyassociated mortality at the end of the twentieth century: Massachusetts, 1990-1999. J Am Med Women Assoc. 2002; 57: 140-3.
25. Geller SE, Rosenberg D, Cox SM, Brown ML, Simonson L, Driscoll CA, Kilpatrick SJ. The continuum of maternal morbidity and mortality: factors associated with severity. Am J Obstet Gynecol. 2004; 191: 939-44.

Recebido em 7 de novembro de 2005

Versão final apresentada em 6 de abril de 2006

Aprovado em 27 de abril de 2006 